\title{
Interfaces between a Saturated Aqueous Urea Solution and Crystalline Urea: A Molecular Dynamics Study
}

\author{
E. S. Boek, W. J. Briels, and D. Feil \\ Chemical Physics Laboratory, University of Twente, P.O. Box 217, 7500 AE Enschede, The Netherlands
}

Received: August 13, 1993; In Final Form: November 9, $1993^{\circ}$

Interfaces between a saturated urea solution and crystalline urea surfaces have been studied by means of molecular dynamics simulations. Mean densities of solute urea and water at both (001) and (110) interfaces show an enhanced adsorption probability for solute urea compared to water. Orientation distributions and positions in the horizontal plane have been calculated for urea molecules in the first adsorption layer. These indicate a "preorganization" for growth of a new crystal layer at the (001) interface. The adsorbed urea molecules at the (110) interface, on the other hand, are much more randomly distributed and oriented than at the (001) interface and even show a slight preference for wrong positions above the crystal surface. This indicates an increased interfacial entropy which may stabilize the (110) crystal surface.

\section{Introduction}

Information about the crystal-aqueous solution interface is essential to gain understanding of crystallization processes at the molecular level. Experimentally, structural details of molecular adsorption on a crystalline surface can be obtained for monolayer coverages. ${ }^{1}$ The interface between a bulk solution and a crystal face, however, is hard to access by direct measurement, as it is surrounded by two dense phases. Computer simulations can play a significant role in this respect, as they provide molecular-level information. We stress that we do not simulate the crystal growth process itself: apart from not being very informative, this would also be unfeasible using molecular dynamics techniques. Instead, we want to investigate those parameters which are important for understanding this process. Interesting microscopic quantities of the interface include among others density profiles, positional and orientational order of the adsorbed molecules, and diffusion coefficients.

Several molecular dynamics and Monte Carlo simulations of solid-liquid interfaces have been published in the last decades. These include hard sphere fluids near hard flat walls, ${ }^{2-4}$ LennardJones crystal-melt systems, ${ }^{5-11}$ interfaces between ice and water, ${ }^{12}$ platinum surfaces and water, 13,14 water at a hydrophobic surface, ${ }^{15}$ a $\mathrm{NaCl}$ crystal and water, ${ }^{16}$ and membrane-aqueous solution interfaces. ${ }^{17,18}$ The general picture emerging from simulations of aqueous systems is a strongly increased liquid density at the interface, lateral positional and orientational ordering in the adsorption layer, and a decreased mobility as compared to the bulk liquid.

Recently, a molecular dynamics study of interfaces between crystalline urea and water was reported. ${ }^{19}$ The structure and dynamics of water molecules at the interface appeared to depend strongly on the structure of the crystal surface. We found that the adsorption of water on the (111) crystal surface was much stronger than on the ( $\overline{1} \overline{1} \overline{1})$ interface. Assuming that preferential solvent adsorption leads to a reduction of the growth rate of a crystal face, ${ }^{20}$ the occurrence of only the (111) surface on the crystal growth form was predicted. For the (001) and (110) interfaces, the adsorption behavior of water alone did not provide sufficient information to explain the experimental habit of urea crystals grown from aqueous solutions. From the crystal surface structures it was conjectured that the randomness and hence the

* To whom correspondence should be addressed at R.I.M. Laboratory of Solid State Chemistry, University of Nijmegen, Toernooiveld, 6525 ED Nijmegen, The Netherlands.

- Abstract published in Advance ACS Abstracts, January 1, 1994. entropy of adsorbed urea molecules may be larger on the (110) surface than on the (001) surface. From this point of view it was inferred that the (110) face would be dominant over the (001) face in the crystal morphology.

In this paper, an MD study is reported on the solution structures near the (001) and (110) interfaces between crystalline urea and a saturated aqueous urea solution. The analysis of the interface structures has been guided by the question of how well the surface and the solution are preorganized to accept a new crystal layer to make the crystal grow.

\section{Molecular Dynamics Simulations}

The molecular dynamics simulations were performed with the GROMOS package. ${ }^{21}$ The computational boxes consisted of a saturated urea solution in contact with two different crystalline urea surfaces. In one simulation the surfaces $(001)$ and its symmetric counterpart $(00 \overline{1})$ were studied, and in a second simulation the symmetric couple (110) and ( $\overline{1} 10)$ was investigated. The saturated urea solution was prepared by putting randomly urea and water molecules in a box in accordance with the desired urea saturation molality of $20 \mathrm{~mol} / \mathrm{kg},{ }^{22}$ which corresponds to a urea mole fraction of 0.265 . A constant $N V T$ equilibration of $10 \mathrm{ps}$ at high temperature $(798 \mathrm{~K})$ was performed to remove any spurious structure in the solution, followed by a 10-ps $N V T$ equilibration run at $298 \mathrm{~K}$. Then the solution was brought into contact with the crystal. Next, the crystal-solution box was equilibrated at constant $N p T$ (in order to allow for volume relaxations) for $20 \mathrm{ps}$ at $298 \mathrm{~K}$ and a pressure of $1 \mathrm{~atm}$. The box dimensions parallel to the (001) interface tended to diverge upon longer $N p T$ run times, which destroys the urea tetragonal crystal structure. Therefore we continued at this point with a 10-ps $N V T$ equilibration run at $298 \mathrm{~K}$, followed by $N V T$ production runs of $70 \mathrm{ps}$ for both interfaces.

To improve statistics, three solution boxes with different starting configurations were generated for each interface. The reason for sampling over different boxes was that increasing the simulation time of one box did not remove unrealistic structures in the center of the box. For all these boxes, the above-mentioned procedure was followed.

Periodic boundary conditions were applied in three dimensions, combined with a cutoff distance for nonbonded interactions of $8.5 \AA$. This is justified because simple static lattice calculations with a summation limit of $8.5 \AA$ show that the lattice energy has reached a value of more than $95 \%$ of the total energy in the convergence limit. In order to prevent the interface layers at both sides of the crystal from being able to "feel" each other due 
TABLE 1: Nonbonded Potential Parameters for Urea $(\mathrm{HHL})^{23}$ and Water (SPC/E) ${ }^{25}$

\begin{tabular}{ccccc}
\hline molecule & atom & $\sigma(\AA)$ & $\epsilon(\mathrm{kJ} / \mathrm{mol})$ & $q(\mathrm{e})$ \\
\hline \multirow{2}{*}{ urea } & $\mathrm{O}$ & 3.21 & 0.954 & -0.38 \\
& $\mathrm{C}$ & 4.06 & 0.619 & 0.38 \\
& $\mathrm{~N}$ & 3.93 & 0.699 & -0.83 \\
& $\mathrm{H}$ & 0 & 0 & 0.415 \\
water & $\mathrm{O}$ & 3.17 & 0.650 & -0.8476 \\
& $\mathrm{H}$ & 0 & 0 & 0.4238
\end{tabular}

TABLE 2: Simulation Characteristics: Computational Boxes for the Saturated Solution (1-3) and Pure Water (w) for Both (001) and (110) Interfaces*

\begin{tabular}{|c|c|c|c|c|}
\hline & \multicolumn{4}{|c|}{ box number } \\
\hline & 1 & 2 & 3 & $\mathrm{w}$ \\
\hline \multicolumn{5}{|c|}{ (001) Interface } \\
\hline$x=4 d_{100}$ & 2.297 & 2.281 & 2.303 & 2.309 \\
\hline$y=4 d_{010}$ & 2.303 & 2.333 & 2.293 & 2.291 \\
\hline$z=9 d_{001}+$ solution & 10.241 & 10.781 & 7.588 & 7.629 \\
\hline$U$ (crystal) & 288 & 288 & 288 & 288 \\
\hline$U$ (solution) & 192 & 212 & 106 & \\
\hline$W$ & 533 & 587 & 294 & 570 \\
\hline \multicolumn{5}{|c|}{ (110) Interface } \\
\hline$x=5 d_{001}$ & 2.407 & 2.405 & 2.405 & 2.406 \\
\hline$y=6 d_{110}$ & 2.459 & 2.463 & 2.469 & 2.467 \\
\hline$z=10 d_{110}+$ solution & 7.349 & 7.335 & 7.329 & 6.856 \\
\hline$U$ (crystal) & 300 & 300 & 300 & 300 \\
\hline$U$ (solution) & 124 & 124 & 124 & \\
\hline$W$ & 344 & 344 & 344 & 570 \\
\hline
\end{tabular}

a Dimensions in nanometers; the number of urea molecules in the crystal is represented by $U$ (crystal) and in solution by $U($ sol), and the number of water molecules by $W . d_{h k l}$ is defined as the distance between two lattice planes $(h k l)$.

to long-wavelength correlations perpendicular to the interface, the thicknesses of both the crystal and solution layers in the $z$-direction were chosen to exceed the cutoff distance by factors of 4 .

During the production runs a drift velocity of the center of mass of the simulation boxes was observed of about $0.005 \AA / \mathrm{ps}$, probably due to roundoff errors. Although it is rather small, this drift obscures the analysis of average quantities at the interface. Therefore the coordinate files were corrected for the drift afterward. For the (110) interface a small shear motion of the crystal interface layers, decreasing toward the center of the crystal box, was found. Its magnitude was only a few permille of the average velocity of the urea molecules. Moreover, for a small shear rate it may be expected that the solution will follow the motion of the crystal surface layers. Therefore we assume that the results are still valuable after correction for this artifact.

The force field for urea was constructed from the $\mathrm{HHL}$ potential ${ }^{23}$ for nonbonded interactions and GROMOS covalent parameters. ${ }^{24}$ For water the SPC/E potential ${ }^{25}$ was used. The nonbonded potential parameters for both water and urea are given in Table 1. The problems mentioned above concerning the crystal indicate that there are some inaccuracies in the urea potential which need improvement. It should be realized that the potential was constructed under space group constraints whereas dynamic simulations probe a much larger part of the potential. Nevertheless, we have successfully used this potential to explain the neutron scattering spectrum of aqueous urea solutions. ${ }^{26}$ Therefore we think the main characteristics of the potential are realistic for the saturated solution. Because we are mainly interested in the behavior of the solution at the interface, we trustfully use it here.

Covalent bond distances were constrained using the SHAKE algorithm, ${ }^{27}$ which allows an integration time step of $2 \times 10^{-15}$ s. Coordinates were sampled every 25 time steps. For more information about the simulation boxes we refer to Table 2 . From this table it appears that the $x$ and $y$ values for the boxes $1-3$ are
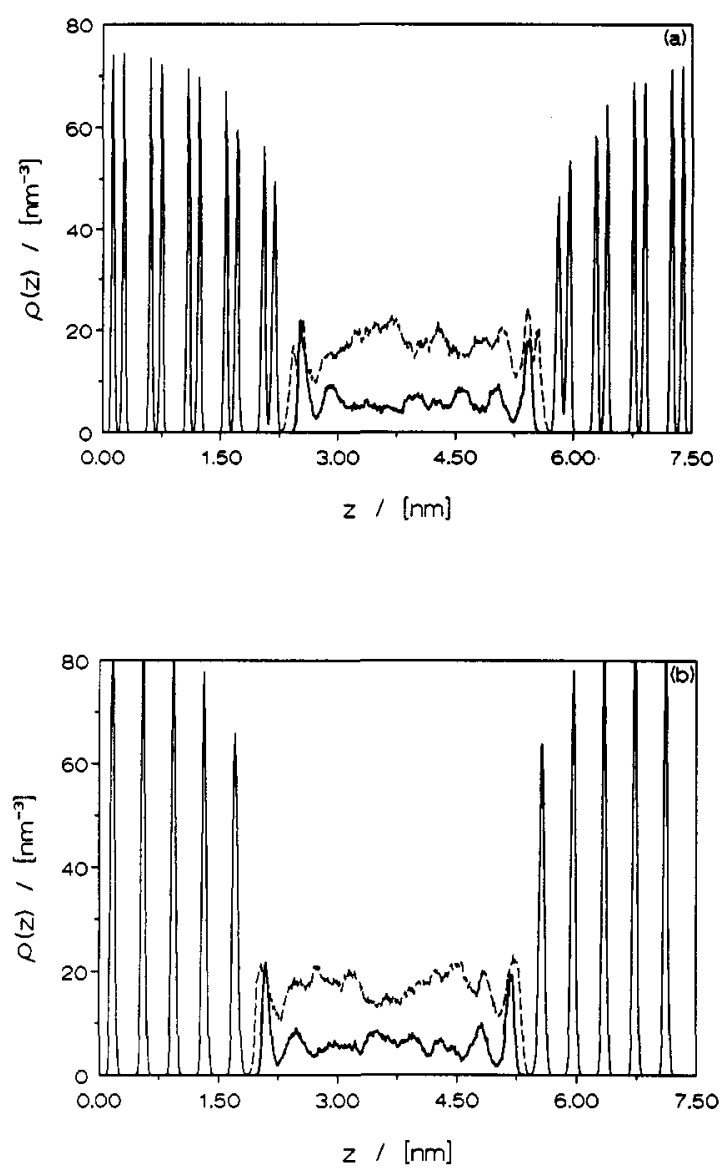

Figure 1. Density profiles of the centers of mass of crystalline urea (solid line), solute urea (fat solid line), and water (dashed line) molecules perpendicular to the two interfaces. Part a shows the (001) surface, and part $b$ the (110) surface.

TABLE 3: Comparison of Urea Thermal Parameters $U_{11}(=$ $\left.U_{22}\right)$ and $U_{33}$ in Units of $10^{-4} \AA^{2}$ from Neutron Diffraction Experiments $^{28}$ (expt) and MD Simulations*

\begin{tabular}{lrrrrc}
\hline atom & expt & bulk & center & surface & surface/center \\
\hline & & & $U_{11}$ & & \\
$\mathrm{C}$ & 353 & 617 & 809 & 1574 & 1.95 \\
$\mathrm{O}$ & 506 & 951 & 1182 & 2112 & 1.79 \\
$\mathrm{~N}$ & 692 & 809 & 978 & 2577 & 2.63 \\
$\mathrm{H}_{1}$ & 838 & 1201 & 1364 & 2616 & 1.92 \\
$\mathrm{H}_{2}$ & 853 & 1056 & 1216 & 4938 & 4.06 \\
& & & $U_{33}$ & & \\
$\mathrm{C}$ & 155 & 258 & 295 & 558 & 1.89 \\
$\mathrm{O}$ & 160 & 259 & 295 & 655 & 2.22 \\
$\mathrm{~N}$ & 251 & 401 & 435 & 876 & 2.01 \\
$\mathrm{H}_{1}$ & 478 & 725 & 758 & 1250 & 1.65 \\
$\mathrm{H}_{2}$ & 267 & 390 & 453 & 1204 & 2.66
\end{tabular}

${ }^{a}$ The simulations include an infinite bulk urea crystal ${ }^{19}$ (bulk) as well as a central (001) layer (center) and a surface (001) layer (surface) of a urea crystal bounded by a saturated urea solution.

slightly different. This is probably due to the instability of the crystal lattice mentioned above.

The total simulation time was approximately $650 \mathrm{CPU}$ hours on a Convex $\mathrm{C} 240$ vector computer.

\section{Solution Structure at the Interface}

Density Profiles. The density profiles of the centers of mass of crystalline urea, solute urea, and water molecules perpendicular to the (001) and (110) interfaces are presented in Figure $1 \mathrm{a}$ and $1 \mathrm{~b}$, respectively. Both interfaces show thermal broadening of the crystal surface layer peaks. The thermal parameters of the crystal surface layers, calculated from rms fluctuations of atom coordinates, are about 2 times larger than for layers in the center of 

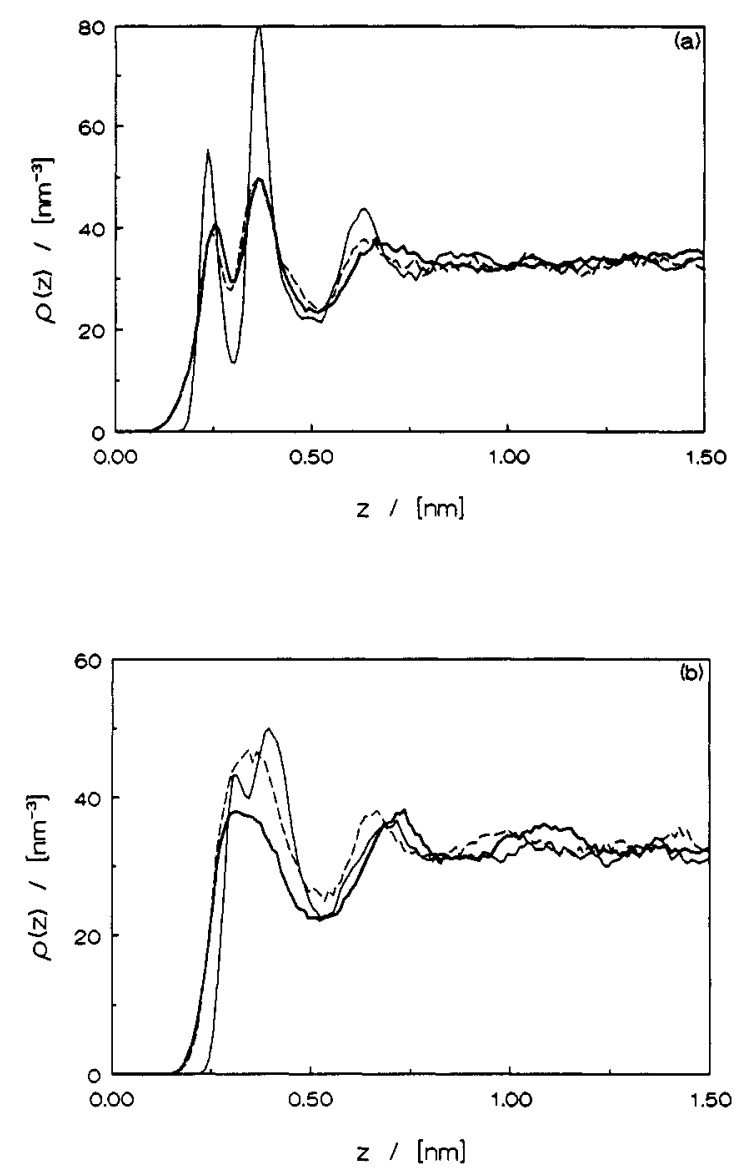

Figure 2. Water density profiles perpendicular to the (001) interface (a) and the (110) interface (b) for three different simulations: "static" (solid line), "dynamic" (dashed line), and "saturated" (fat solid line).

the crystal. These central layers can be considered as real bulk layers, because their thermal parameters are similar to those of an infinite urea crystal;:19 see Table 3.

The ratios of the mean square displacements in the surface layer compared to the central crystal layer are shown in the last column of Table 3. These ratios are roughly the same parallel $\left(U_{11}\right)$ and perpendicular $\left(U_{33}\right)$ to the surface. This may be due to the small compressibility of the liquid. For $\mathrm{H}_{2}$ this ratio is considerably higher than for all other atoms, which is due to the fact that the $\mathrm{H}_{2}$ atom protrudes into the liquid. The influence of the colliding liquid molecules is transferred to the other atoms of the surface urea molecules only indirectly. Therefore this ratio decreases for the atoms lying deeper in the crystal, going from $\mathrm{H}_{2}, \mathrm{~N}, \mathrm{H}_{1}$, and $\mathrm{C}$ to $\mathrm{O}$. The positions of the atoms in the urea molecule have been indicated in Figure 7.

The simulated bulk parameters are rather high compared to the experimental ones. ${ }^{28}$ We suggest that the real characteristics of the solid-solution interface are bounded by the two extremes of the static crystal ${ }^{19}$ and this dynamic crystal. In other words, we can be sure that any structure that survives this thermal motion will certainly be present.

Now we will concentrate on the solution. From Figure 1, in which the results for a single box are shown, it appears that both solute urea and water molecules show long-wavelength density fluctuations up to the center of the solution box. These do not vanish upon longer simulation times. Therefore the density profiles, measured as a function of the distance to the center of mass of the outermost crystal surface layers, were averaged for all three boxes. Furthermore, the density profiles in Figure 1 are not symmetrical. This is probably due to the slowness of the dynamics of the adsorbed solution layer. Therefore the left and right parts of the solution boxes were averaged, which is allowed due to the crystal symmetry. The resulting solution profiles for

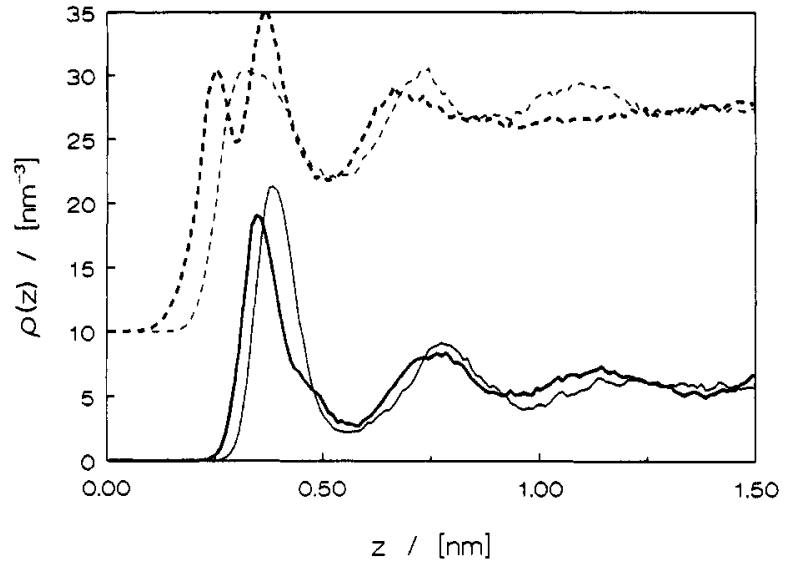

Figure 3. Density profiles of the saturated urea solution: Solute urea at the (001) interface (fat solid line) and the (110) interface (solid line); water at the interfaces (001) (fat dashed line) and (110) (dashed line), translated by 10 units on the $y$-axis for clarity.

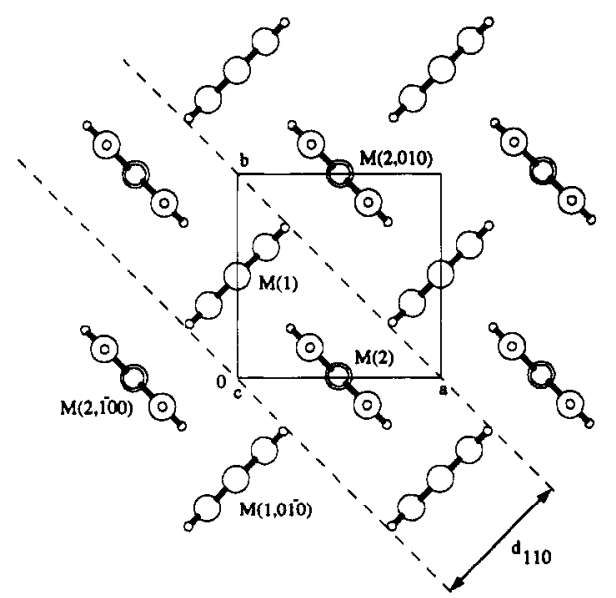

Figure 4. Projection of the urea crystal structure perpendicular to the (001) surface. A cross section of the crystal surface layer of the (110) interface has been indicated by the symbol $\mathrm{d}_{110}$.

TABLE 4: Interface Adsorption of Solute Urea and Water: Urea Mole Fractions (Umf) and Mean Densities of the First Adsorption Layer for Solute Urea $\left(\overline{\rho_{\mathrm{U}}}\right)$ and Water $\left(\overline{\rho_{\mathrm{W}}}\right)$ Relative to the Bulk Urea and Water Densities, Respectively

\begin{tabular}{cccc}
\hline interface & Umf & $\overline{\rho_{\mathrm{U}}}$ & $\overline{\rho_{\mathrm{W}}}$ \\
\hline$(001)$ & 0.31 & 1.23 & 0.66 \\
$(110)$ & 0.35 & 1.42 & 0.68
\end{tabular}

water and urea, which are presented in Figures 2 and 3, respectively, show that most of the density fluctuations have vanished, except for some clear adsorption layers.

We will consider water and solute urea separately now.

Water. In our paper on water near a rigid urea crystal ${ }^{19}$ we have conjectured that (1) the dynamics of the crystal will only smear the height and the position of the water adsorption peaks at the static crystal but leave the main characteristics unaffected, and (2) the presence of solute urea in the solution will hardly influence the water distribution.

In order to verify these conjectures, the density profiles at the $(001)$ and (110) interfaces are compared in Figure 2a and $2 b$, respectively, for three different simulations: (1) "static"-pure water at a rigid urea crystal; ${ }^{19}$ (2) "dynamic" - pure water at a dynamic urea crystal; (3) "saturated" - water in a saturated urea solution at a dynamic urea crystal (this paper). The details of the latter two simulations are presented in Table 2.

For the (001) interface both assumptions seem to hold true. The density profile for "dynamic" water shows two smeared-out adsorption peaks at the same $z$-distance as the one for "static" water. The "saturated" profile, scaled up to the pure water density, 


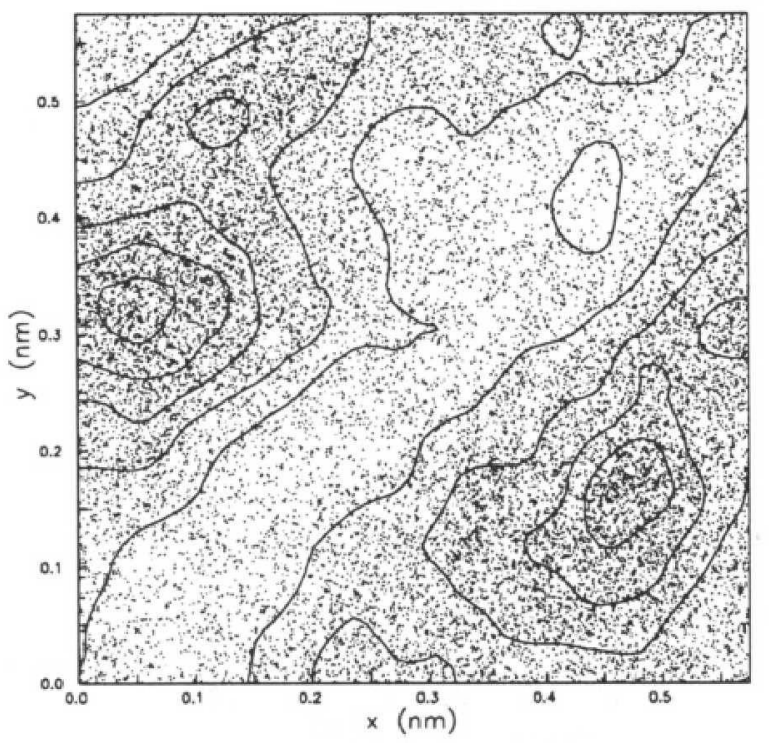

(a)

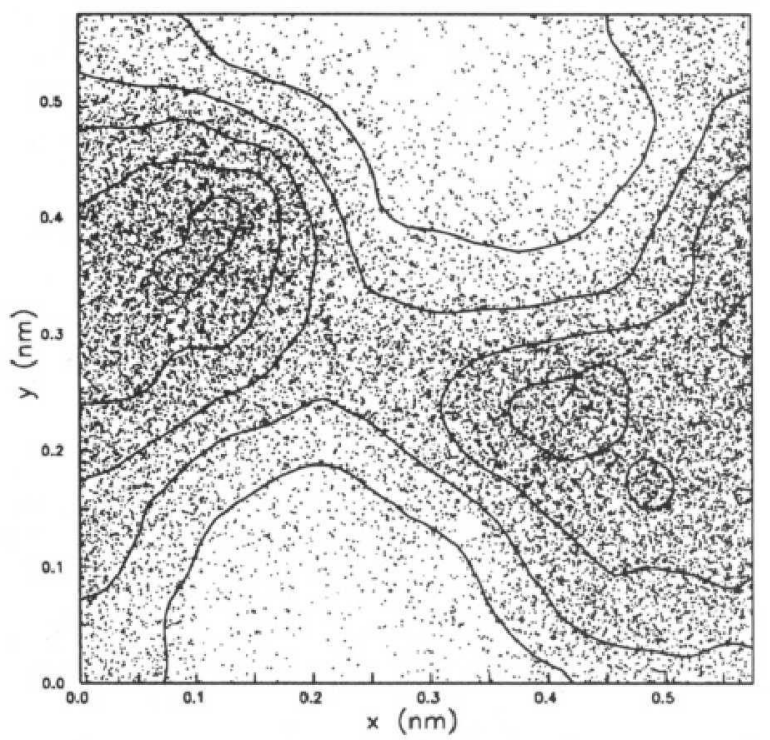

(b)

Figure 5. Positional distribution of the solution molecules in the first adsorption layer as a function of their positions along the (001) surface: (a) solute urea, (b) water.

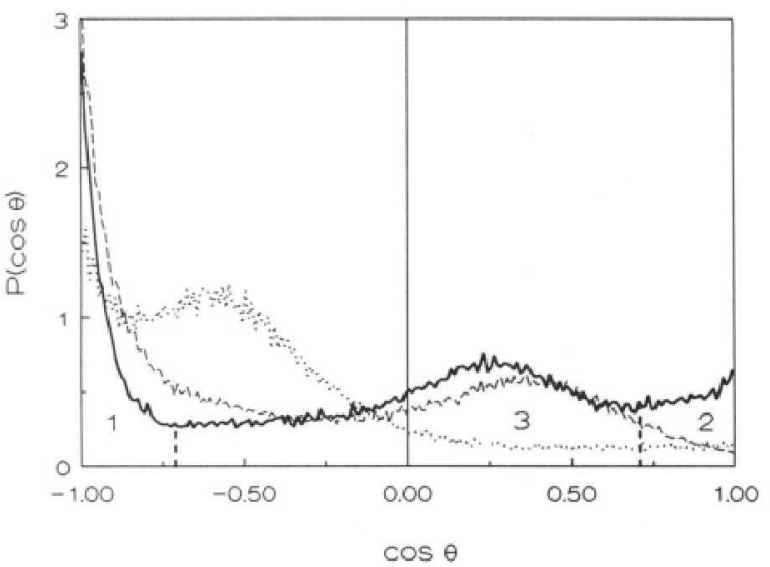

Figure 6. Orientational distributions at the (001) interface of the dipole vectors of solute urea (fat solid line) and water (dotted line) and the water $\mathrm{OH}$ vectors (dashed line) as a function of $\cos \theta(\theta$ is the angle between the mentioned vectors and the outward surface normal). Three different regions are discerned: (1) $\cos \theta(\mathrm{O}-\mathrm{C})<-0.7$; (2) $\cos \theta(\mathrm{O}-\mathrm{C})$ $>0.7 ;(3)-0.7<\cos \theta(\mathrm{O}-\mathrm{C})<0.7$.

is almost identical to the "dynamic" one: this means that the positional correlations of the water structure in the $z$-direction are unaffected by the presence of solute urea.

For the (110) interface, the same arguments hold to a lesser extent: the two weakly separated "static" water layers fuse into one peak in the "dynamic" profile. Moreover, the water density in the "saturated" adsorption peak (scaled up to pure water) is lower than in the "dynamic" one: apparently solute urea effectively "chases away" water in the adsorption layer.

As both assumptions seem to be more or less justified, we suggest that the adsorption phenomena at the other interesting urea interfaces (111) and ( $\overline{1} \overline{1} \overline{1})$ remain qualitatively the same upon simulation of a saturated solution at a dynamic crystal. If this holds true, water adsorption at the (111) interface is so strong that it will "poison" and thus retard the growth of this crystal face. $^{19}$

Solute Urea. In Figure 3 the density profiles of solute urea are shown. For comparison, those of water are also included in this figure. At both interfaces two well-defined urea peaks occur, corresponding to two adsorption layers. The first peak at the (001) interface peak reveals a shoulder at $0.47 \mathrm{~nm}$. The origin

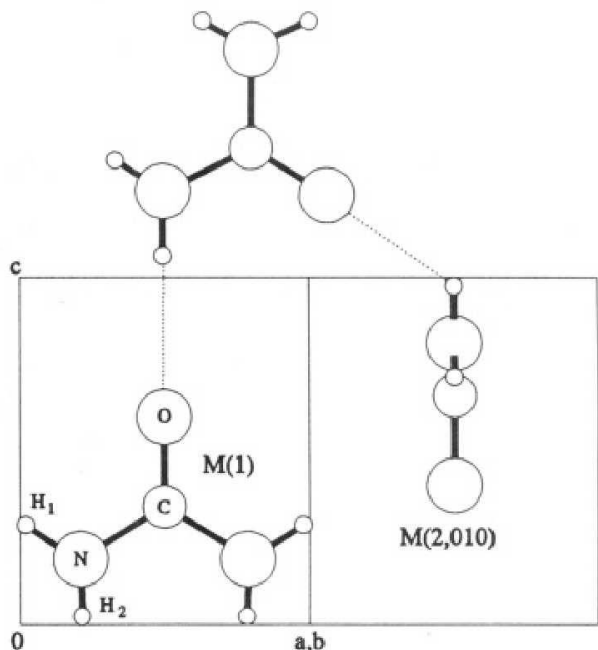

Figure 7. Formation of hydrogen bonds along the $[\overline{1} \overline{1} 0]$ direction between a solute urea molecule and the urea crystal molecules $\mathrm{M}(1)$ and $\mathrm{M}(2,010)$. A structure projection along [1 110$]$ of one crystal unit cell at the $(001)$ interface is drawn.

of this shoulder will be explained in the following section. It is remarkable that the adsorption profiles show very similar features for both interfaces: the heights of the first peaks are similar, whereas the positions in the $z$-direction of these peaks approximately correspond to the distance to a next crystal layer in each case. It seems that the positional distribution of urea in the $z$-direction is much less sensitive to the actual surface geometry than the one for water.

The mean densities of water and solute urea in the first adsorption layer, measured up to the first density minimum (and the second minimum in the case of water at the (001) interface) are presented in Table 4. For both interfaces they show an increase for urea and a decrease for water relative to the bulk density. The urea mole fraction in this layer is increased for the (001) and (110) interfaces by factors of 1.2 and 1.3 , respectively, compared to those for the bulk solution (Table 4). This indicates an enhanced adsorption probability for urea compared to water.

Positional and Orientational Distributions along the Interface. For both interfaces we have calculated the distribution of the solution molecules in the first adsorption layer as a function of 


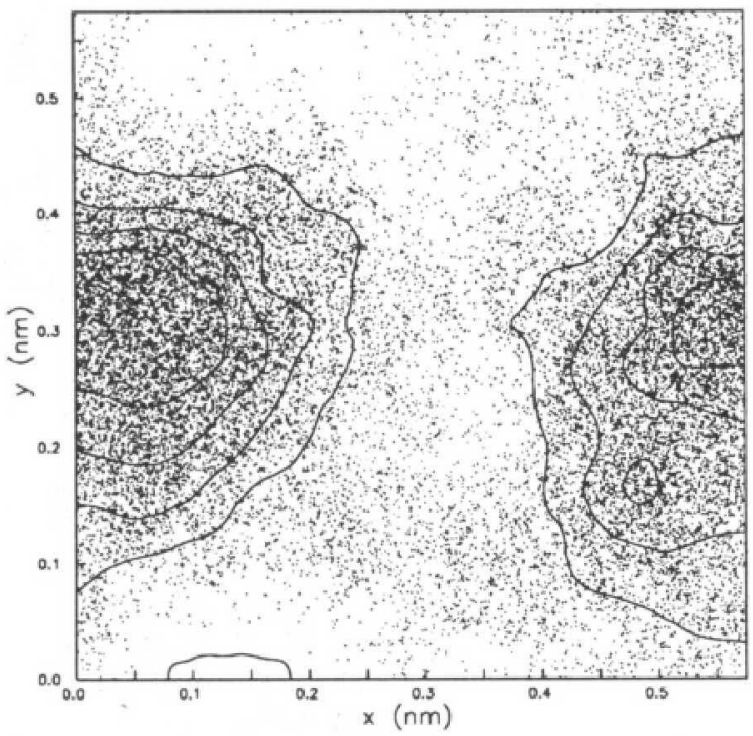

(a)

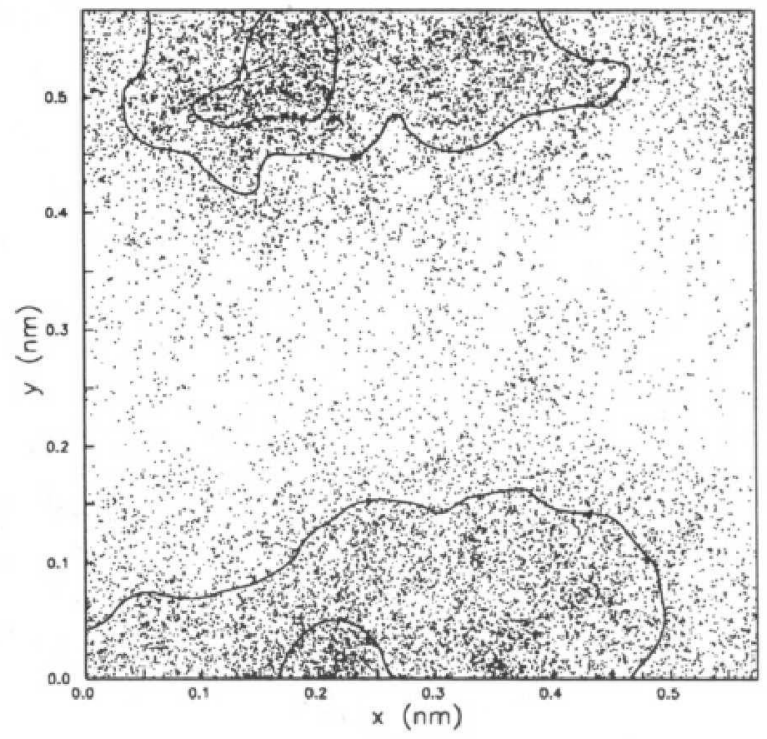

(b)

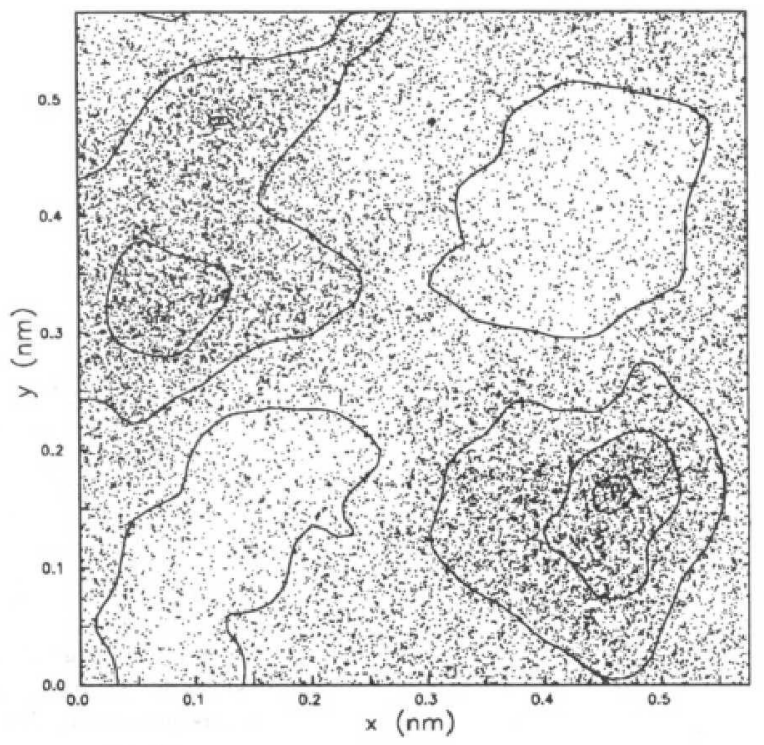

(c)

Figure 8. Positional distribution of the centers of mass of solute urea molecules adsorbed on the (001) surface, split up according to (a) cos $\theta(\mathrm{O}-\mathrm{C}$ ) $<-0.7$, (b) $\cos \theta(\mathrm{O}-\mathrm{C})>0.7$, and (c) $-0.7<\cos \theta(\mathrm{O}-\mathrm{C})<0.7$.

their position along the surface. In order to judge how well the adsorbed urea molecules are "prepared" to form the next crystal layer, we have determined the correlation between their positions and orientations with respect to the crystal surface molecules. In the description of the results we will refer only to the left interface (with respect to the positions in Figure 1). In all cases the results of both the left and the right surface were averaged by applying appropriate transformations to the coordinates of the molecules near the latter surface.

In the following, the urea crystal axes are referred to as $a, b$, and $c$, whereas the axes of the simulation boxes are indicated as $x, y$, and $z$. For the (001) interface these axes frames coincide; for the (110) interface, we refer to Table 2.

The (001) Interface. In Figure 4 a structure projection perpendicular to the (001) crystal surface (along [001]) is presented for one unit cell. Urea crystallizes in the tetragonal space group $\mathrm{P} \overline{4}{ }_{1} \mathrm{~m}(a=b=0.566 \mathrm{~nm}, c=0.471 \mathrm{~nm})$. The unit cell contains two molecules: molecule $\mathrm{M}(1)$ centered at the fractional coordinates $(0,1 / 2, z)$ and molecule $M(2)$ at $(1 / 2,0$, $1-z$ ), with $z \simeq 0.32$. This means that there is a "hole" in the surface above molecule $\mathrm{M}(1)$, when it is viewed along the $c$-axis. This molecule has a downward-pointing $\mathrm{O}-\mathrm{C}$ (dipole) vector, while the higher positioned molecule $\mathrm{M}(2)$ has an upward-pointing $\mathrm{O}-\mathrm{C}$ vector.

The positions of the centers of mass of the solute urea and water molecules in the first adsorption layer, transformed to one unit cell using crystal surface periodicity, are shown in Figure 5a and $5 \mathrm{~b}$, respectively. We will consider the water molecules first. The water distribution displays maxima in the positions above $\mathrm{M}(1)$ and $\mathrm{M}(1,100)$ (i.e. $\mathrm{M}(1)$ translated over one period in the $a$-direction) and closely follows the contours of the hole. The distributions of the water dipole and $\mathrm{O}-\mathrm{H}$ vectors (Figure 6) 


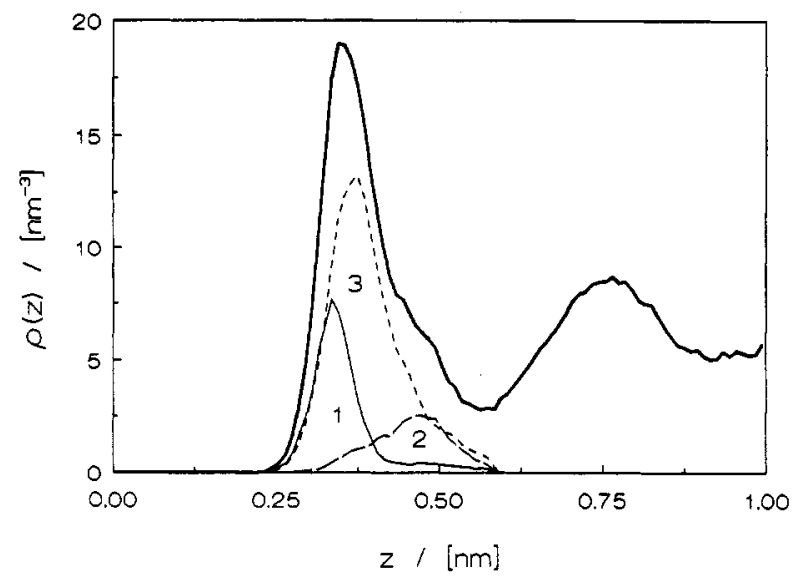

Figure 9. Density profile of solute urea adsorbed on the (001) surface (fat solid line), split up according to (1) $\cos \theta(\mathrm{O}-\mathrm{C})<-0.7$ (solid line), (2) $\cos \theta(O-C)>0.7$ (long-dashed line), and $(3)-0.7<\cos \theta(O-C)<$ 0.7 (short-dashed line).

show patterns similar to those obtained from our previous calculations: ${ }^{19}$ the water points with one $\mathrm{O}-\mathrm{H}$ bond into the surface. There is also a new feature: the strongest peak in the water dipole distribution is located at $\cos \theta \approx-1$, which may imply that it aligns with the urea $\mathrm{M}(1)$ dipole.

Now we will turn to solute urea. The center of mass distribution (Figure 5a) shows a remarkably high density around the positions $(3 / 4,1 / 4)$ and $(1 / 4,3 / 4)$ in fractional coordinates. This can be explained by the fact that a solute urea molecule can form an attractive hydrogen bond between the upward-pointing oxygen of $\mathbf{M}(1)$ and the upward-pointing $\mathrm{NH}_{2}$ group of $\mathbf{M}(2,010)$, as can be seen from Figure 7. Between $M(1)$ and $M(2)$ such a bond is unlikely on geometric grounds. A second characteristic is an enhanced density above the molecules $M(1)$ and $M(1,100)$. This corresponds to the positions of molecules $M(1)$ which would be present in the next crystal layer in the positive $c$-direction. As a third feature, we mention the relatively high density above the molecules $M(2)$ and $M(2,010)$. These are the positions of $M(2)$ molecules which would be present in the second missing crystal layer. As a result of all three points, there is a low-density zone running from the lower-left to the upper-right part of the box.

The construction proposed in Figure 7 implies a preference of the angle $\theta(\mathrm{O}-\mathrm{C})$ for values of about $70^{\circ}$, where $\theta(\mathrm{O}-\mathrm{C})$ is the angle between the urea dipole vector and the outward-pointing surface normal. Indeed, as shown in Figure $6, \cos \theta(\mathrm{O}-\mathrm{C})$ displays an enhanced probability around the value of 0.3 . Moreover, if the molecules adsorbed above $\mathrm{M}(1)$ and $\mathrm{M}(1,100)$ are to be considered as growth units, i.e. as belonging to the growing crystal, their dipoles should be antiparallel to the outward surface normal. This is confirmed by the strong maximum in the orientational distribution at $\cos \theta(O-C) \approx-1$. Finally we notice the small peak at $\cos \theta(\mathrm{O}-\mathrm{C}) \approx+1$, which we expect to correspond with the molecules in the second "growth layer".

In order to check the correlations between orientational and positional distribution, we have split up the urea dipole orientation distribution into three parts and constructed the corresponding scatter plots along the interface as well as the density profiles along the $c$-direction. The positional distribution for molecules with $\cos \theta(\mathrm{O}-\mathrm{C})<-0.7$ is presented in Figure 8a. It appears indeed that this orientational preference is coupled to the crystal position M(1). Similarly, the distribution of the molecules with $\cos \theta(\mathrm{O}-\mathrm{C})>0.7$, presented in Figure $8 \mathrm{~b}$, shows a correlation with the crystal molecule position $\mathrm{M}(2)$, which can also be explained by dipole aligning. The split-up density profiles in Figure 9 confirm this picture: the solute peak correlated to the hole above $\mathrm{M}(1)$ is located at a distance of $0.33 \mathrm{~nm}$ from the crystal surface layer, which would be exactly the position of the future crystal layer $M(1,001)$. It is sharply separated from the solute urea above $\mathrm{M}(2)$ at a distance of $0.47 \mathrm{~nm}$, i.e. the place

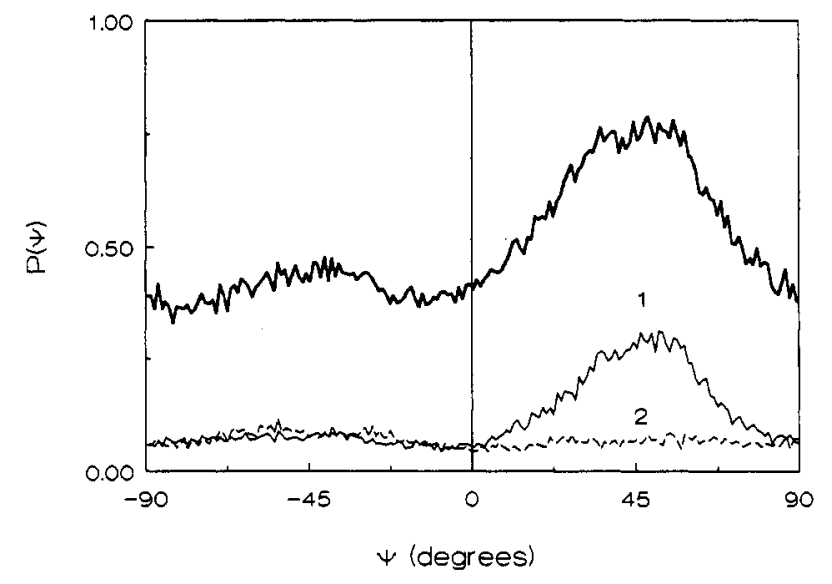

Figure 10. Probability distribution of the angle $\phi(\mathrm{N}-\mathrm{N})$ between the $\mathrm{N}-\mathrm{N}$ vectors of solute urea adsorbed on the $(001)$ surface and the surface $a$-axis (fat solid line), split up according to (1) $\cos \theta(\mathrm{O}-\mathrm{C})<-0.7$ (solid line) and (2) $\cos \theta(\mathrm{O}-\mathrm{C})>0.7$ (dashed line).

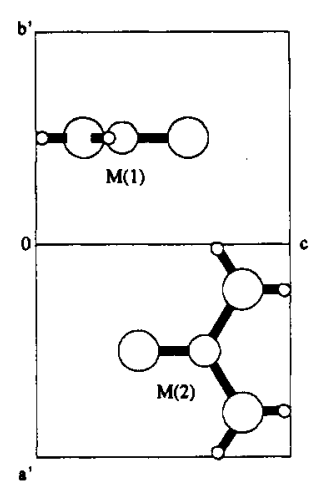

(a)

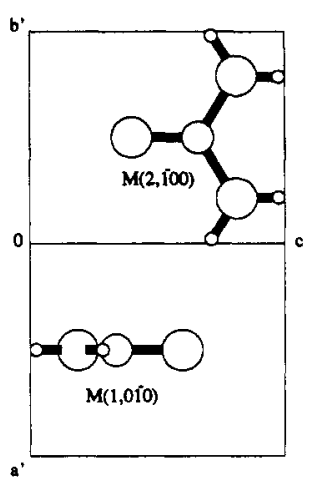

(b)
Figure 11. Projections of the urea crystal structure perpendicular to the (110) surface along [1]10]: (a) surface layer; (b) next "growth" layer along [110].

of the following growth layer $\mathrm{M}(2,001)$ at one interplanar lattice spacing $d_{001}$. This latter peak causes the shoulder in the first urea adsorption peak. Finally, the positional distribution of the molecules with $-0.7<\cos \theta(\mathrm{O}-\mathrm{C})<0.7$, shown in Figure 8c, displays a preference for the positions $(3 / 4,1 / 4)$ and $(1 / 4,3 / 4)$.

As a last test we calculated the distribution of the angle $\phi(N-$ $\mathrm{N})$ between the urea $\mathrm{N}-\mathrm{N}$ vector and the surface $a$-axis for these three layers (Figure 10). The first layer contributes mostly to the maximum at $\phi=45^{\circ}$, again corresponding to the crystal M(1) geometry (see also Figure 4).

We may summarize our results by saying that the first adsorption peak at the (001) interface is kind of "preorganized" to form the first and second growth layers.

The (110) Interface. In Figure 4, a cross section fo the crystal surface layer of the (110) interface is indicated. In Figure 11a, a structure projection perpendicular to this surface layer along [1'10] is shown for one unit cell. A future crystal layer in the direction of the solution (along [110], see Figure 4) will then consist of $M(2, \overline{100})$ and $M(1,0 \overline{10})$, which is shown in Figure $11 \mathrm{~b}$.

In Figure $12 \mathrm{a}$ and $12 \mathrm{~b}$ the distributions of the adsorbed solute urea and water molecules in the horizontal plane are presented. For urea the adsorption pattern is quite diffuse compared to the one at the (001) interface: the difference between the highest and lowest contour lines is 5 times smaller. Only in the upperleft and lower-right corners of the unit cell, corresponding to the positions above $M(1)$ and $M(2)$ in the "old" surface layer, can some "blobs" of solute urea be distinguished. Apparently, the adsorbed urea molecules have a preference for a wrong position from a crystal growth point of view. Of the two blobs, the one in the lower-right corner is the more intense. This can be understood by noticing that it corresponds to an attractive position 


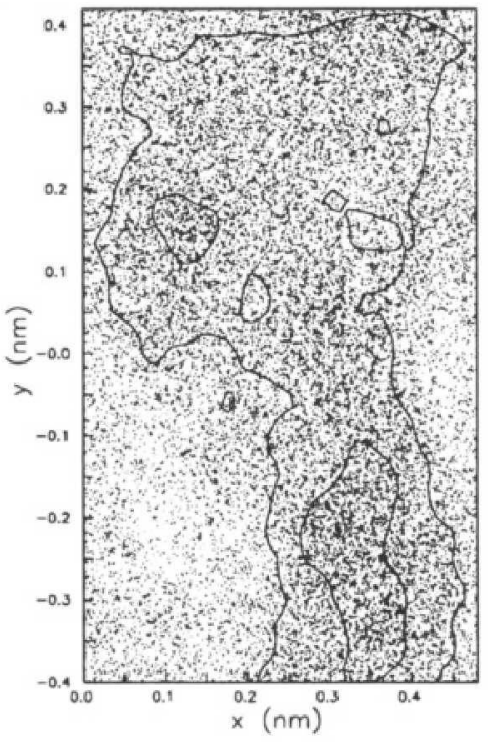

(a)

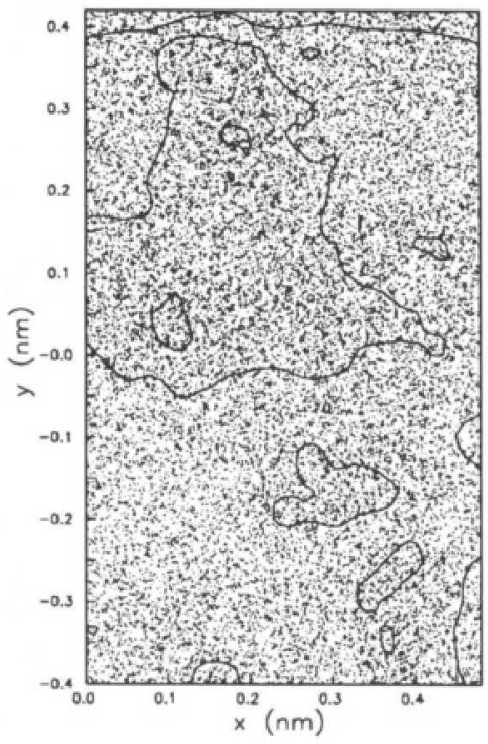

(b)

Figure 12. Positional distribution of the solution molecules in the first adsorption layer as a function of their position along the (110) surface: (a) solute urea; (b) water.

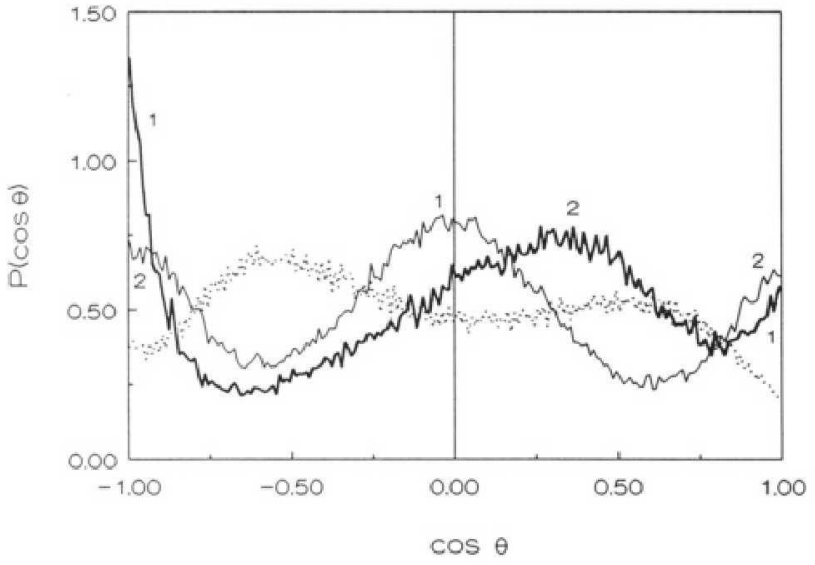

Figure 13. Orientational distribution at the (110) interface of the dipole vectors of solute urea (fat solid line) and water (dotted line) and the urea $\mathrm{N}-\mathrm{N}$ vectors (solid line) as a function of $\cos \theta(\theta$ is the angle between the mentioned vectors and the outward surface normal).

above the flat-lying molecule $\mathrm{M}(2)$. For water the adsorption profile is even more chaotic.

In order to obtain more insight into the orientation of the molecules, the probability distributions of the water and urea dipoles and urea $\mathrm{N}-\mathrm{N}$ vectors were calculated as a function of $\cos \theta, \theta$ being the angle between these vectors and the outward surface normal (Figure 13). It is striking that the urea dipole distribution is similar to the one at the (001) interface, except for the fact that the amplitudes at the (110) interface are much smaller. Maxima in this distribution are found for dipoles antiparallel to the outward surface normal $(\cos \theta(\mathrm{O}-\mathrm{C}) \approx-1)$ and for dipoles parallel to this normal $(\cos \theta(\mathrm{O}-\mathrm{C}) \approx 1)$. These maxima are labeled with a " 1 " in Figure 13. The $\mathrm{N}-\mathrm{N}$ vectors of these molecules are expected to be parallel to the surface. Indeed there is a maximum in the $\cos \theta(\mathrm{N}-\mathrm{N})$ distribution function around zero, which is labeled with a " 1 " as well. It will be clear that the other maximum in the distribution of $\cos \theta(\mathrm{O}-\mathrm{C})$ around 0.35 is coupled to the maxima in $\cos \theta(\mathrm{N}-\mathrm{N})$ around 1 and -1 . These are labeled with a "2" in Figure 13. This implies a preference for urea molecules having dipoles almost parallel to the surface with $\mathrm{N}-\mathrm{N}$ vectors perpendicular to the surface.

Both orientations (" 1 " and " 2 ") are unfavorable for the crystallization of a new growth layer. In order to investigate whether these molecules can poison the (110) interface by hydrogen bonding to the crystal surface, the probability distributions of the urea dipole and $\mathrm{N}-\mathrm{N}$ vectors as a function of the angle $\phi$ were calculated. Here $\phi$ is the angle between these vectors and the surface $y$ and $x$ axes, respectively. A completely flat distribution over the angle $\phi$ was found for both vectors (not shown). Hence the possibility of a poisoning of this surface by hydrogen bonding can be neglected. On the other hand, the flat $\phi$ distribution hints at some kind of entropic poisoning.

From all this we may conclude that the positional distribution in the horizontal plane and the orientational correlations for the (110) interface are very poor compared to those for the (001) interface.

\section{Discussion and Conclusions}

The structure of interfaces between crystalline urea and saturated aqueous urea solutions has been studied by means of MD simulations. The features of the water density profiles at the interface do not seem to be very sensitive to the dynamics of the urea crystal or the presence of solute urea in the solution.

The density profiles for solute urea perpendicular to the crystal surface are very similar for both the (001) and the (110) interfaces. The position of the first adsorption peak corresponds to one crystal layer spacing in each case. The mean urea density in the first layer is considerably higher than the bulk solute urea density. For water the opposite holds true. This indicates that urea is more strongly adsorbed than water.

The distributions in the horizontal plane of the urea molecules in the first adsorption layer are different for the two interfaces. The (001) interface shows a preference for a positional and orientational distribution corresponding to a new crystal growth layer. A shoulder in the first adsorption peak could be related to a second growth layer. The distribution is too diffuse to call the adsorbed molecules a new crystal layer, but certainly a sort of "preorganization" for crystal growth is present at this interface.

In the $\mathrm{BCF}$ theory ${ }^{29}$ it is assumed that crystal growth from slightly supersaturated solutions will occur according to a spiral growth mechanism, whereas at higher supersaturations a twodimensional nucleation mechanism ("birth and spread") will become dominant. The present study of the (001) interface, however, indicates that even for a saturated solution a twodimensional nucleation mechanism may be operating, as the structure of the adsorbed solute urea molecules suggests formation of crystallization nuclei on the crystal surface. It may even be 
that kinetic roughening has started; this would be in accordance with the very slow surface diffusion we expect. All this will lead to an enhanced growth rate or even disappearance of this face from the crystal growth form.

The (110) interface shows a totally different picture. The positional and orientational distributions are much more diffuse than at the (001) interface, whereas the adsorbed urea molecules show a preference for wrong positions above the crystal surface. Our results indicate that the randomness of solute urea molecules at the (110) interface is larger than at the (001) interface. As a result, it is quite improbable that the adsorbed molecules collectively position and orient themselves in such a way that a new crystal layer can be formed. This effect may be less dramatic at existing steps or kinks. In thermodynamic terms one may say ${ }^{19}$ that the interfacial entropy is quite large at the (110) surface, which stabilizes the crystal face. This will lead to a reduction of the growth rate for this crystal face.

We conclude that the habit of urea crystals grown from aqueous solution, showing a small (001) surface and a large (110) surface, ${ }^{30}$ can qualitatively be understood from urea adsorption phenomena at the crystal-solution interface. The actual surface structure has played a crucial role in obtaining these results, and we think detailed molecular models of the interface are indispensible for future studies of the morphology of molecular crystals. We are convinced that many important concepts related to crystal growth cannot be described by the traditional Kossel-type models, which are based on units of equidimensional blocks rather than on real molecules.

In order to obtain quantitative results for the growth rates of both crystal surfaces, potentials of mean force for adsorption and surface diffusion of solute urea molecules at the interface have to be calculated. MD simulations on this subject are in progress.

Acknowledgment. E.S.B. is grateful to the Netherlands Foundation for Scientific Research (SON/NWO) for financial support.

\section{References and Notes}

(1) Thiel, P. A.; Madey, T. E. Surf. Sci: Rep. 1987, 7, 211

(2) Snook, I. K.; Henderson, D. J. Chem. Phys. 1978, 68, 2134

(3) Henderson, J. R.; van Swol, F. Mol. Phys. 1984, 51, 991.

(4) Groot, R. D.; Faber, N. M.; van der Eerden, J. P. Mol. Phys. 1987, 62,861 .

(5) Toxvaerd, S.; Prastgaard, E. J. Chem. Phys. 1977, 67, 5291

(6) Snook, I.; van Megen, W. J. Chem. Phys. 1979, 70, 3099.

(7) Toxvaerd, S. J. Chem. Phys. 1981, 74, 1998.

(8) Broughton, J. Q.; Bonissent, A.; Abraham, F. F. J. Chem. Phys. $1981,74,4029$.

(9) Broughton, J. Q.; Gilmer, G. H. J. Chem. Phys. 1983, 79, 5095

(10) Broughton, J. Q.; Gilmer, G. H. J. Chem. Phys. 1986, 84, 5749.

(11) Broughton, J. Q.; Gilmer, G. H. J. Chem. Phys. 1986, 84, 5759.

(12) Karim, O. A.; Haymet, A. D. J. J. Chem. Phys. 1988, 89, 6889.

(13) Spohr, E. J. Phys. Chem. 1989, 93, 6171.

(14) Raghavan, K.; Foster, K.; Motakabbir, K.; Berkowitz, M. J. Chem. Phys. 1991, 94, 2110.

(15) Lee, C. Y.; McCammon, J. A.; Rossky, P. J. J. Chem. Phys. 1984, $80,4448$.

(16) Anastasiou, N.; Fincham, D.; Singer, K. J. Chem. Soc., Faraday Trans. 2 1983, 79, 1639.

(17) Schlenkrich, M.; Nicklas, K.; Brickmann, J.; Bopp, P. Ber. BunsenGes. Phys. Chem. 1990, 94, 133.

(18) Nicklas, K.; Böcker, J.; Schlenkrich, M.; Brickmann, J.; Bopp, P. Biophys. J. 1991, 60, 261.

(19) Boek, E. S.; Briels, W. J.; van Eerden, J.; Feil, D. J. Chem. Phys. $1992,96,7010$.

(20) Berkovitch-Yellin, Z. J. Am. Chem. Soc. 1985, 107, 8239.

(21) van Gunsteren, W. F.; Berendsen, H. J. C. Groningen Molecular Simulation (GROMOS) Library; Biomos: Groningen, 1987.

(22) Ellerton, H. D.; Dunlop, P. J. J. Phys. Chem. 1966, 70, 1831.

(23) Hagler, A. T.; Huler, E.; Lifson, S. J. Am. Chem. Soc. 1976, 96 , 5319.

(24) Hermans, J.; Berendsen, H. J. C.; van Gunsteren, W. F.; Postma, J. P. M. Biopolymers 1984, 23, 1513.

(25) Berendsen, H. J. C.; Grigera, J. R.; Straatsma, T. P. J. Phys. Chem.

$1987,91,6269$

(26) Boek, E. S.; Briels, W. J. J. Chem. Phys. 1993, 98, 1422.

(27) van Gunsteren, W. F.; Berendsen, H. J. C. Mol. Phys. 1977, 34, 1311 .

(28) Guth, H.; Heger, G.; Klein, S.; Treutmann, W.; Scheringer, S. Z. Kristallogr. 1980, $153,237$.

(29) Burton, W. K.; Cabrera, N.; Frank, F. C. Philos. Trans. R. Soc. London, $A$ 1951, 243, 299.

(30) Davey, R.; Fila, W.; Garside, J. J. Cryst. Growth 1986, 79, 607. 